Arm training in Multiple Sclerosis using Phantom: clinical relevance of robotic outcome measures

Peer-reviewed author version

FEYS, Peter; ALDERS, Geert; GIJBELS, Domien; DE BOECK, Joan; DE WEYER, Tom; CONINX, Karin; RAYMAEKERS, Chris; TRUYENS, Veronik; Groenen, Patric; Meijer, Kenneth; Savelberg, Hans \& OP 'T EIJNDE, Bert (2009) Arm training in Multiple Sclerosis using Phantom: clinical relevance of robotic outcome measures. In: 11th International Conference on Rehabilitation Robotics (ICORR 2009). p. 576-581..

Handle: http://hdl.handle.net/1942/10360 


\title{
Arm training in Multiple Sclerosis using Phantom: clinical relevance of robotic outcome measures
}

\author{
Feys Peter ${ }^{1}$, Alders Geert ${ }^{1}$, Gijbels Domien ${ }^{1}$, De Boeck Joan², De Weyer Tom², Coninx Karin ${ }^{2}$, \\ Raymaekers Chris ${ }^{2}$, Truyens Veronik ${ }^{3}$, Groenen Patric ${ }^{3}$, Meijer Kenneth ${ }^{4}$, Savelberg Hans ${ }^{4}$, Eijnde O \\ Bert $^{1}$
}

\begin{abstract}
Upper limb weakness due to Multiple Sclerosis has a major negative effect on the functional activities of the patient. Promising developments in the field of rehabilitation robotics may enable additional exercise. This study aims to investigate which types of robotic outcome measures are clinically relevant, in preparation of the evaluation for intervention studies. Within this context, appropriate movement tasks and tests for the haptic PHANTOM end-effector robot were designed in a virtual environment. These tasks focused on spatial accuracy, object manipulation and speed. Outcome measures were: 1) virtual movement tests, recorded by the robot to quantify motor control; 2) clinical outcome measures such as the Motricity Index, Jamar and MicroFET hand-held dynamometer to evaluate muscle strength; and the Nine Hole Peg Test, Purdue Pegboard, ARAt and TEMPA to asses upper limb function and manual dexterity.10 healthy controls performed the virtual movement tasks using the Phantom as interface. $21 \mathrm{MS}$ subjects with upper limb dysfunction caused by muscle weakness were included in an interventional training study. Pearson correlations were calculated at baseline between the performance on the three virtual movement tasks and the clinical tests on impairment and activity level. The virtual movement tests discriminated between healthy controls and MS patients with hand dysfunction. In the MS patient group, no significant correlations were found between muscle strength tests and virtual movement tasks, while mainly significant correlations were found between specific functional measures (specifically ARAt and Purdue pegboard test) and virtual movement tasks.
\end{abstract}

Manuscript received February 1, 2009.

This work was supported in part by the European Interreg III project (\# 4-BMG-II=84) as well as the Province of Limburg (Belgium). The research part at EDM is funded by EFRO (European Fund for Regional Development), the Flemish Government and the Flemish Interdisciplinary institute for Broadband technology (IBBT).

Peter Feys and Bert O. Eijnde are associate professors at BIOMED/REVAL Rehabilitation \& Health Care Research Center (PHL university college and Uhasselt), Hasselt (Guffenslaan 39, 3500 Hasselt; email: pfeys@mail.phl.be; peter.feys@uhasselt.be, bopteijnde@mail.phl.be and bopteijnde@mail.phl.be). Geert Alders and Domien Gijbels are researchers (galders@mail.phl.be, dgijbels@mail.phl.be).

Karin Coninx is professor, and Chris Raymaekers associate professor at the Hasselt University-tUL-IBBT, Expertise Centre for Digital Media (EDM)

Wetenschapspark 2, 3590 Diepenbeek, Belgium (+32 112684 11,

karin.coninx@uhasselt.be, chris.raymaekers@uhasselt.be). Joan De Boeck is post-doctoral researcher, Tom De Weyer is researcher

(tom.deweyer@uhasselt.be, joan.deboeck@uhasselt.be).

Kenneth Meijer and Hans Savelberg are associate professions at the

Department of Human Movement Science, Faculty of Health, Medicine and Life Sciences, Universiteit Maastricht, Netherlands (+31.43.388.1392, hans.savelberg@bw.unimaas.nl and Kenneth.Meijer@BW.unimaas.nl).

Veronik Truyens is coordinator of paramedical services, and Patric Groenen director in the Rehabilitation and MS Centre Overpelt, $(\mathrm{Tel}+32$ 118091 00, veronik.truyens@msreva.be).

\section{INTRODUCTION}

Multiple Sclerosis (MS) is a chronic, progressive disease of the central nervous system (CNS). Although the exact aetiology of MS remains unknown, a combination of genetic, infectious, environmental and/or autoimmune factors likely contributes to disease onset. Dependent on the heterogeneous distribution of lesions throughout the CNS, MS may clinically lead to a variation of symptoms such as muscle weakness, spasticity, loss of coordination, sensory disorder and also visual and cognitive deficits, which all may cause severe limitations of functioning in daily life. MS predominantly affects young adults in their most productive years. Its prevalence in Europe varies with latitude with an average being approximately 1/1000.

For a long time, persons with MS have been advised to avoid physical exercise, as it was believed that this would increase risk for MS relapses. At present, however, an increasing number of studies have shown beneficial effects of exercise training in MS regarding lower limb muscle strength, exercise tolerance level, functional mobility (i.e. balance and walking) and quality of life, while no evidence of deleterious effects were described [1,2]. Unfortunately, the outcomes of exercise therapy on arm function in MS have hardly been investigated. This is surprising because upper extremity dysfunction strongly influences the capacity to perform activities of daily life (ADL) such as self-care, dressing, object manipulations, etc.

Training duration and intensity are considered to be key factors for a successful neurological rehabilitation[3]. As therapy time dedicated to arm function training is limited with MS persons having a multiplicity of symptoms requiring treatment, there is need for additional therapeutic modalities that can be used autonomously by the patients. Within this framework, rehabilitation robotics are new promising developments allowing high-intensity, repetitive, task-specific, interactive treatment of the impaired upper limb. Haptic interactive arm robotic systems provide proprioceptive and visual feedback during the performance of goal-directed movements in a virtual learning environment.

The effects of robot-aided therapy on arm motor performance, functional capacity and movement quality in persons with MS are still completely unknown. In stroke, a recent systematic review revealed a small effect of upper 
limb robotic training on motor function but not ADL (activities of daily life) function[4]. Kwakkel et al. pointed, however, that many clinical trials did not include valid clinical scales that measure clinical dexterity of the arm or hand. Besides, the ADL scales included may not have been able to reflect true recovery.

In this study, appropriate virtual movement tasks and tests were designed for upper extremity training in a virtual environment using an existing haptic end-effector device called PHANTOM. The aim of the present study was to evaluate the relationship between robotic (movement and motor control) related outcome measures with clinically valid measures for hand and arm dexterity. The present study is part of a larger pilot intervention study investigating, in these 21 persons with MS, the effects of 4 weeks robot-aided arm training on motor control and clinical outcome measures.

\section{METHODS}

A) PHANTOM haptic device and virtual movement tasks including outcome measures

A PHANTOM 1.5 haptic device (SensAble Technologies)[5], controlled by 3HD, was selected as robotic training interface. This device was used before in rehabilitation context $[6,7]$. It was handled through a penlike stylus allowing tracking of the stylus tip position in 6 degrees-of-freedom (DoF), as well as providing force feedback in 3 DoF (translational). The PHANTOM was coupled to an interactive virtual learning environment (see below), so that robotic tasks and tests can be executed while visual feedback is provided. In order to provide the patients with a large enough working field, the end-effector Phantom was used in combination with a 19" monitor. As such, unrestricted movements of shoulder (flexion and extension, abduction and adduction, inwards and outwards rotation), elbow (flexion and extension, pronation and supination) and wrist (flexion and extension, ulnar and radial deviation) joints were possibly involved during training. The stimulation of whole-limb movement control during training is dependent on individual capacities. Therefore, gain could be personally regulated, whereby large and small respectively stands for large/small ranges of stylus/upper limb motion that had to be executed.

Data were logged at an average sampling frequency of $1000 \mathrm{~Hz}$. Force, position, orientation, velocity and contact/collision reaction forces are recorded as a vector in coordination frame ( $\mathrm{x}, \mathrm{y}, \mathrm{z}$ attributes).

During robot-assisted training, MS subjects were instructed to manipulate the PHANTOM stylus in order to control an object in a virtual environment. In this pilot study, a physical or occupational therapist administered each robotic therapy session, ensuring proper positioning of the patient in front of the Phantom and screen as well as giving instructions when needed and if necessary to intervene in emergency situations. An adapted virtual learning environment had been designed by EDM in close collaboration with the REVAL Research Group of the PHL
University College. Three different robot tasks and tests are applied to train and evaluate MS subjects on arm movement performance (e.g. spatial accuracy, positional stability and maximal velocity).

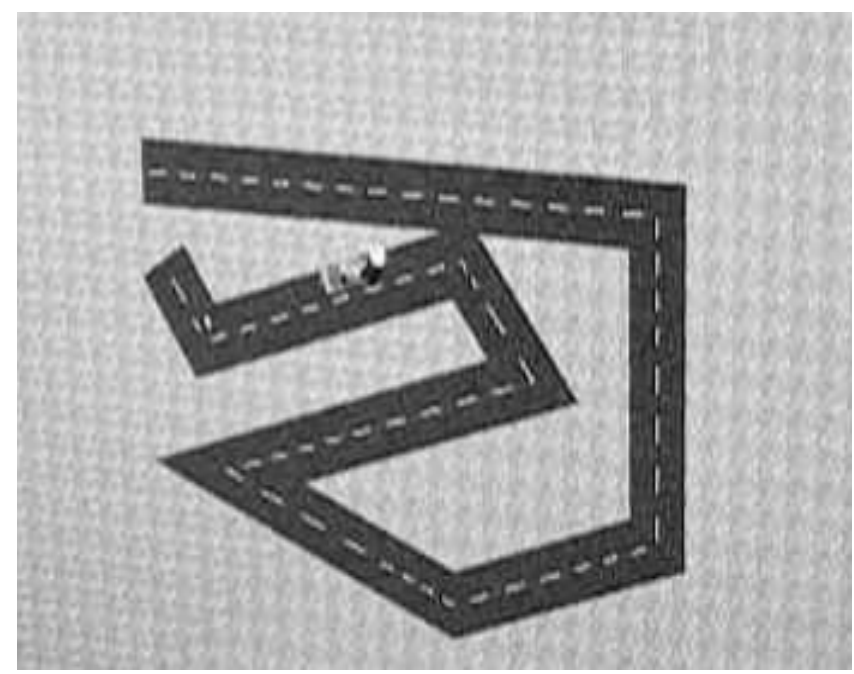

Figure 1. Illustration of the car trajectory task.

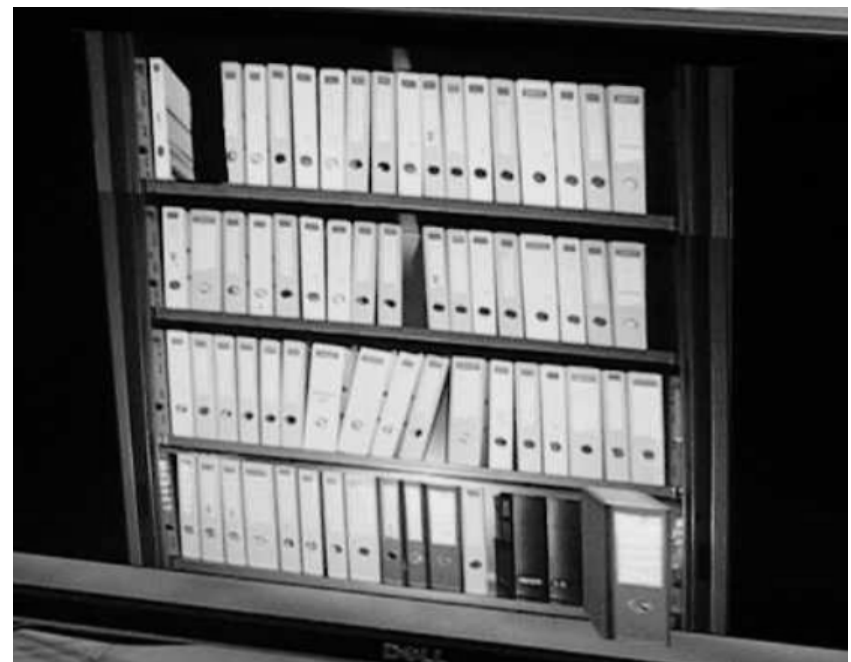

Figure 2. Illustration of the object manipulation task.

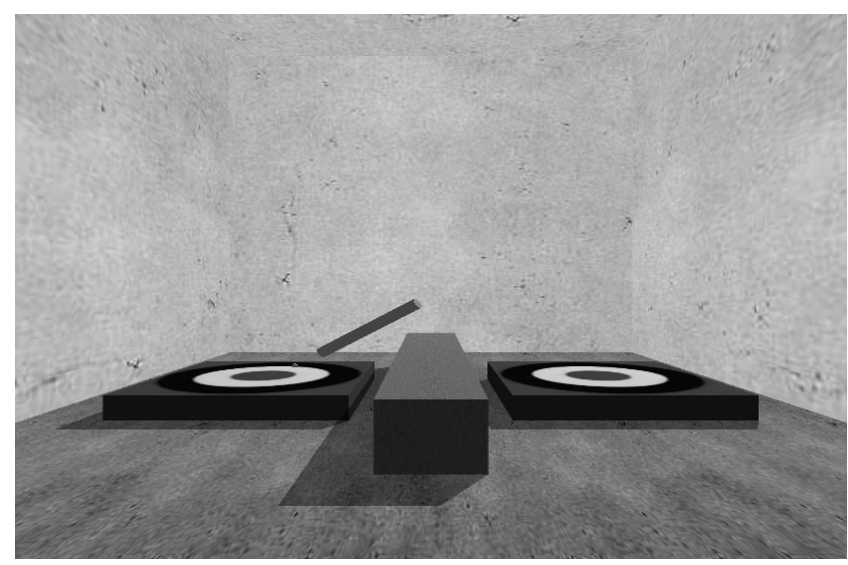

Figure 3. Illustration of the speeded tapping task. 
To allow patients with different disabilities to perform tasks successfully or to allow for progressive training, parameters could be personally adapted. Three types of movement tasks were developed in which virtual objects were moved in a virtual stationary environment: a car trajectory tasking requiring motor accuracy, an object manipulation task requiring major pro-supination control similarly to daily life activities such as pouring water, and a speeded movement tasks. As such, we believe that a relatively wide range of arm movements and motor control functions were included. During the car trajectory task, subjects had to operate the stylus of the PHANTOM to pilot a car throughout a predefined pathway (see illustration in Figure 1). The task was developed to train the subject on spatial accuracy and to stimulate, amongst others, pro- and supination movements by including curves in the required trajectory. Force feedback could be applied to help the subject to keep the car on the required track. Dependent on the level of motor control of the patient, this force can be set on small, medium or large, with 'small' allowing the patient to leave the track and 'large' force feedback being used to resists patient's movements when leaving the track (or making errors). Finally the implementation of viscosity, which exerts an opposite force to the movement direction, could also be added so that subjects had to produce more strength to accomplish the task.

With the object manipulation task (see Figure 2), the subject needs to virtually grab a book by positioning the stylus there for two seconds. Further, the goal of this task was to put the book in a predefined place in a closet. The available

space in the closet to position the book can be small or wide (movement accuracy), can differ in location (high/low, left/right) and can be oblique (again forcing the patient to control pro- and supination of the forearm) in order to make the task more difficult. Moreover, the weight of the book could be adjusted (up to $3 \mathrm{~kg}$ ) to provide proprioceptive feedback and to increase the motor control demands.

The speeded tapping task (see illustration in Figure 3) was inspired on the real-life plate tapping task (see below). The aim of this virtual version of plate tapping was to speeded movements between two targets (=plates). A contact with the plate is called a tap. As many correct taps as possible have to be made in a 30 second time interval. Correct means that no collision occurs with the obstacle, and that taps are on the plates.

Of each task, various variations were programmed (f.e. other car trajectory, different place of the book) in order to avoid subjects to be bored by repetitiveness. For testing, one standard variation of each task was identified. Robotic outcome measures on the tests were time needed to execute the task, distance or trajectory covered during the task and maximal speed performance (in the speeded tapping task). The third execution of the robotic tests were used as a learning effect had been showed before[8].

\section{B) Participants and clinical outcome measures}

All MS patients $(n=21,13$ female and 8 male, mean age $59,7 \pm 11$ years) were selected from the Rehabilitation and MS centre Overpelt (Belgium). All subjects were submitted to a standard neurological examination by a neurologist. The inclusion criteria were a definite clinical diagnosis of MS and upper limb dysfunction caused by muscle weakness. Subjects were excluded when there had been a relapse of MS or treatment with glucosteroids in the last month prior to the study, presence of upper limb tremor, upper limb paralysis or severe cognitive or visual disability interfering with the interaction with the robotic device. The mean disease duration for this group was 23,37 years $( \pm 16,31)$. 20 Subjects were right-handed. Preferably, the dominant hand of the participants was tested when eligible, which was the case in 8 participants.

In addition to the patient group, a healthy control group ( $n=10,4$ female and 6 male, mean age 48,0 \pm 6,5 years) was included for comparison and consisted of staff working in the centre. The tested hand on the Phantom robot for this group was the dominant hand for half of the subjects and the non-dominant hand for the other half. All subjects were right handed. The study was approved by the local Ethical committee of the Rehabilitation and MS centre Overpelt, as well as the ethical committee of the University of Hasselt. All subjects have given their written consent before they participated in the study.

The clinical assessment consisted of measurements for muscle strength as well as hand and arm functionality.

A calibrated digital MicroFET2 and electronic JAMAR hand-held dynamometers (Biometrics, Gwent, UK) were used to quantify muscle strength. A hand-held dynamometer is a reliable assessment instrument when practiced by a single experienced tester. Reference values for extremity muscle strength obtained by a hand-held dynamometer from healthy adults aged 20 to 79 are available[9]. The MicroFET2 was used to measure isometric force (expressed in $\mathrm{Nm}$ ) for a number of upper-extremity muscle actions. These were shoulder (flexion, extension, exo- and endorotation, abduction), elbow (flexion, extension) and forearm movements (pronation and supination). The JAMAR handgrip test was used to determine hand force (expressed in Nm). Standardized instructions and positioning were followed. The subject was seated with the arm next to the body and the elbow in $90^{\circ}$ flexion. The person was asked to squeeze as hard as possible. The test was repeated three times and the mean of the results was used as outcome of the grip strength. Another clinical strength test was the Motricity Index. Originally the test involves measures for the upper and lower limb. In this study only the 3 items to evaluate the upper limb were used. This upper limb evaluation was based on 3 assessments: Pinch grip (using a 2,5 cm cube between the thumb and the forefinger), Elbow flexion (arm touches shoulder), Shoulder abduction (moving flexed elbow from off the chest). Each item is scored between 0-33, while 1 is added to the total sum (maximal strength is 100). 
Functional capacity of the upper limb was evaluated by the Nine Hole Peg Test, the Purdue Pegboard Test, the Action Research Arm test and the TEMPA.

The 9 Hole Peg Test (9HPT) is a quantitative measure of arm and hand function. It has been used with increasing frequency in clinical practice and in MS clinical trials while reliability and validity have been shown[10]. Subjects were instructed to insert the pegs one by one into the 9 holes and then to remove these pegs one by one. The time needed (seconds) to place and remove all 9 pegs is measured. The Purdue Pegboard Test (PPT) is designed to test hand dexterity. Specifically, it tests hand movement and fingertip dexterity. The PPT is a sufficiently reliable assessment to use with persons with multiple sclerosis. In this study the subject was instructed to insert as many pins as possible in the pegboard in 30 seconds time; unilateral performance was measured. The Action Research Arm test (ARAt) was constructed and found reliable for assessing recovery of upper extremity function (focal disability) following stroke(16). The ARAt is designed to evaluate both hand sides of the subjects, in order to obtain a more total description of the upper extremity function. The ARAt consists of four subscales - grasp, grip, pinch and gross movement - comprising 19 items in total giving a maximum score of 56. The TEMPA (Test d'Evaluation de la performance des Membres Supérieurs des Personnes Agées) has been shown to be a valid test to evaluate the functionality of the upper limb in persons with MS[11]. The TEMPA is composed of nine standardized tasks that simulate activities of daily life. The outcome parameter used in this study was speed of execution (seconds). Only the 4 unilateral tasks were tested given that the robotic evaluation was unilateral: pick up and move a jar, pick up a pitcher and pour water into a glass, handle coins, pick up and move small objects. In the results section, these tasks will be named as "Jar", "Pouring water", "Handle coins" and "Moving small objects" respectively. Plate tapping test is a test of limb movement speed. It is a subtest of the Eurofit Test Battery[12]. While tapping the subject is seated in front of the plate, the other hand has to rest in the middle of the tapping-board. The plates used in this test were $60 \mathrm{~cm}$ apart. The subject was asked to tap with the tested hand on these two plates during 30 seconds and to perform as many taps as possible within this timeframe. For the second test the subject was asked to perform 20 taps as fast as possible during which time was recorded.

\section{C) Experimental design and data-analysis}

After user tests to assess feasibility and clinical acceptance of working with the PHANTOM in the virtual learning environment, a single-centre cross-over study design is being performed. The final goal is to assess the effects of a 4-week training program with the newly designed robotic movement tasks on the arm motor performance and functional capacity in persons with MS. We report here on 1) the performance of health controls and MS patients on the robotic movement tests and 2) the relation between performance on the robotic movement tests and the clinical measurements of upper limb muscle strength and arm functionality in persons with upper limb dysfunction.

Mean and standard deviation of the upper limb strength tests and measures of functionality were calculated to describe the performance of the subjects. One-way ANOVA was performed to determine the difference between virtual robotic task performance for the MS group and healthy controls. Pearson correlation coefficients were calculated to examine, for the MS group only, the relation between performance on the robotic movement tests and the clinical strength and functional outcome measures. It must be noted that, for the timed functionality tests, subjects were only included in the analysis when they were able to execute the specific test. As such, correlation coefficients were mostly calculated on 18 subjects. All analyses were conducted using Statistica 6.0 software (Statsoft, Tulsa, USA).

\section{RESULTS}

First, the performance of the MS group on the virtual movements tasks were compared to that of the healthy control group (see table 1). The performance of a healthy control and MS case is illustrated in figure 4.

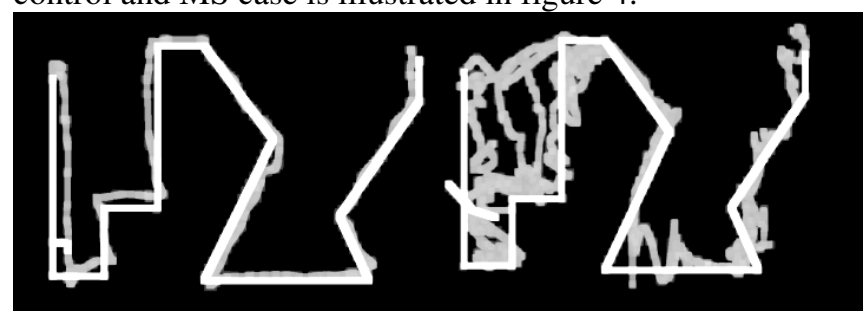

Figure 4. Car Trajectory task performed by a healthy control (left) and a person with arm dysfunction due to MS (right).

ANOVA analyses revealed a significant difference for time performances on all type of tasks, with MS patients with upper limb dysfunction being slower than healthy controls. Surprisingly, only a tendency towards significance was found for the spatial measures (total distance or trajectory covered) for the car trajectory task. Distance measures were across tasks larger for the MS group compared to the control group indicating on a decreased motor control.

Table 1. Outcome measures of the virtual movement tasks for the MS group and Healthy controls.

\begin{tabular}{|c|c|c|c|c|c|}
\hline & \multicolumn{2}{|c|}{ MS GROUP } & \multicolumn{2}{|c|}{ HEALTHY CONTROLS } & \multirow[b]{2}{*}{$\mathrm{p}$} \\
\hline Car Trajectory & Mean & Std.Dev. & Mean & Std.Dev. & \\
\hline Total time $(\mathrm{s})^{*}$ & 56,663 & 35,169 & 33,236 & 7,140 & 0,048 \\
\hline Total distance & 4,124 & 2,332 & 2,678 & 0,337 & 0,063 \\
\hline \multicolumn{6}{|c|}{ Object Manipulation } \\
\hline Total time $(\mathrm{s})^{*}$ & 38,946 & 28,472 & 11,312 & 5,322 & 0,005 \\
\hline Total distance ${ }^{*}$ & 5,771 & 3,419 & 2,767 & 0,602 & 0,011 \\
\hline \multicolumn{6}{|c|}{ Speeded Tapping } \\
\hline Total distance* & 14,376 & 9,061 & 31,038 & 12,546 & 0,000 \\
\hline Avg Velocity* & 0,526 & 0,275 & 1,032 & 0,401 & 0,000 \\
\hline $\mathrm{Nr}$ Correct taps ${ }^{*}$ & 23,100 & 20,295 & 56,200 & 11,419 & 0,000 \\
\hline Nr Wrong taps & 6,850 & 5,019 & 12,300 & 11,879 & 0,086 \\
\hline
\end{tabular}


No significant correlation was found for the relationship between any muscle strength test with performance on the virtual movement tasks.

A few significant correlations were found in the MS patient group between virtual robotic task performance and functional outcome measures for the MS group (see table 2). The 9HPT, measuring hand dexterity, did not show any significant correlation with robotic outcome measures. In contrast, the Purdue Pegboard test also evaluation hand dexterity (but more difficult) does correlate well with the robotic outcome measures except for the total distance of the car trajectory task. It appears that the relationship between performance on virtual robotic tasks and the ARAt correlates in a better way than with the other arm functional tests. In fact, all temporal and spatial robotic variables were significantly correlated with its total score except for the averaged speed during the speeded tapping task. Correlations can be considered as moderate to high. Only two items of the TEMPA, 'pouring water' and 'manipulating objects', correlated with mainly one virtual task, i.e. Plate Tapping.

Table 2. Pearson correlation coefficients between performance on virtual movement tests (spatial: distance/trajectory and temporal: time) and tests for arm functionality in the MS patient group.

\begin{tabular}{|c|c|c|c|c|c|c|c|}
\hline TASKS & Traje & tory & Object $\mathrm{m}$ & ipulation & Spe & eded tap & ing \\
\hline MEASURES & Distance & Total Time & Distance & Total Time & Total Time & Avg Velocity & Correct Taps \\
\hline 9HPT (s) & ns & ns & ns & ns & ns & ns & ns \\
\hline Purdue Pegboard (s) & $\mathrm{ns}$ & $-0,56$ & $-0,63$ & $-0,51$ & 0,62 & 0,56 & 0,64 \\
\hline ARAt & $-0,48$ & $-0,61$ & $-0,69$ & $-0,56$ & 0,51 & ns & 0,49 \\
\hline TEMPA jar (s) & $\mathrm{ns}$ & ns & ns & ns & ns & $-0,48$ & ns \\
\hline TEMPA water $(\mathrm{s})$ & ns & ns & ns & ns & $-0,54$ & $-0,50$ & ns \\
\hline TEMPA coins (s) & ns & ns & ns & ns & $\mathrm{ns}$ & ns & ns \\
\hline TEMPA objects (s) & $\mathrm{ns}$ & ns & 0,61 & ns & $-0,56$ & $-0,53$ & ns \\
\hline TEMPA total (s) & $\mathrm{ns}$ & ns & ns & $\mathrm{ns}$ & ns & $\mathrm{ns}$ & $\mathrm{ns}$ \\
\hline Plate tapping & ns & $-0,49$ & $-0,58$ & ns & 0,77 & 0,76 & 0,71 \\
\hline
\end{tabular}

\section{DISCUSSION}

This pilot study investigated 1) the discriminative value of virtual movement tasks, performed via an end-effector haptic robot, between healthy controls and persons with arm dysfunction due to MS, and 2) in the MS patient group, its relationship between upper limb muscle weakness, hand and arm functionality, and performance. It appeared that MS patients performed significantly worse than healthy controls regarding the virtual movement tasks while its relationship with traditional functionality measures performance was rather low, except for the Purdue Peg Board test and the Action research arm test.

In this pilot study, a haptic robotic device was used in integration with a newly constructed virtual environment. The manipulation of the stylus of the robotic Phantom device was feasible for all selected patients, although it must be noted that the size of the thin stylus was made larger to allow easier hand grip for patients with marked muscle paresis. Three types of movement tasks were developed with the aim to include motor control parameters (spatial accuracy, positional stability and speed variation) that are important during ecological movements. As well, haptic feedback was added for both proprioceptive feedback as well as a tool for individualization of the tasks to different levels of disability and changes in performance levels. The newly designed virtual tasks to facilitate repetitive practice and evaluation of different types of movement control were well received by the MS patients. It is however noteworthy that the threedimensional book/object manipulation task was initially experienced as more difficult, and later most challenging task. This task requires positional stability of the stylus during a given moment of time, in order to grasp the object. Furthermore, an unique and precise end-position in a 3D environment is required for accurate placement of the book. Given the 3D component in this task, it may be that mild visual dysfunction (although it is noted that nobody reported interfering visual dysfunction) or difficulties in visuomotor transformation had a significant impact on patient's performance, besides differences in muscle weakness. This paper reports on a first analysis of data collected at baseline of a larger interventional cross-over study with the robotic training system.

The healthy controls performed better than the MS patient sample, indicating that the virtual movement tasks were susceptible to detect impaired movement control due to muscle weakness. Surprisingly, during the car trajectory task, movement inefficiency as reflected by increased trajectory was only borderline significant. This may be related to the fact that decreased spatial movement control could also have led to a smaller trajectory made by patients, because of not following the outer corners of the required track. Another explanation could be that patients with muscle weakness and decreased motor control benefited from the force feedback that was provided during the car trajectory task in order to assist patients to keep on track. In other words, aberrant arm movements were smoothly redirected to required track, possibly explaining the relative small performance differences between healthy controls and patients. Finally, one could argue that differences between groups reported above may relate to hand dominance, as only 8 of $21 \mathrm{MS}$ patients performed the task with their dominant hand. This factor cannot be fully excluded, however, one should realize that patients with upper limb dysfunction may change hand dominance over time when the previously dominant arm is more impaired than the previously non-dominant arm. Furthermore, inspection of raw data did not yield indications that reported hand dominance differentiated results in the MS patient group.

The results of this pilot study did, in the patient group, not show any correlation of severity of muscle weakness and robotic performance what was unexpected. This indicates that patients with severe muscle paresis may still be able to perform the virtual tasks quite well due to compensatory movements of for example the trunk (instead of shoulder abduction movement), or by another hand grip of the stylus. As the Phantom is an end-effector type of robot, which is easy to use in a clinical setting, it is acknowledged that the quality of the executed movements is not objectively 
documented. Together with a consortium partner, we aim to measure the quality of movement performed before, during and after training by means of accelerometric devices.

Only few functional tests showed a significant correlation with the performance on the virtual movement tasks. The ARAt (ordinal scale) as well as the Purdue peg board test related quite systematically with the robotic outcome measures, in contrast compared to purely timed movement tasks such as the items of the TEMPA as well as the Nine Hole Peg test (continuous scale). The ARAt was also advised as a candidate to measure arm functionality in stroke, what is confirmed here by our data in MS[4]. The low correlations of the TEMPA (both on item level as total score) were rather disappointing and may indicate that motor function/control measured by a robotic device during a virtual task is better reflecting arm functional capacity rather than ADL. It is acknowledged that no perfect correlations between arm function in daily life and performance with the robot were expected given that manipulation of objects in real environment require a much higher degree of fine dexterity to grasp, stabilize and move an object. Intriguingly, the speeded tapping task measuring repeated maximal speed production (acceleration and deceleration) did relate to items such as pouring water and moving objects.

\section{CONCLUSION}

It is concluded that arm functional capacity, measured with the ARAt and Purdue Peg Board test, is relatively well related to movement control performance during a virtual movement task while activities measured with the TEMPA are not. As such, it seems that motor control being measured using robotic movement tasks is related to arm motor function and functional capacity, however, not to activities of daily life.

\section{ACKNOWLEDGMENT}

The authors acknowledge the contribution of Mia Thijs and Niels Goyens (Rehabilitation and MS centre, Overpelt) and Julie Raedschelders (REVAL) in clinical testing and providing clinical feedback). The authors thank the voluntarily participation of the patients to the study.

\section{REFERENCES}

[1] R. W. Motl and J. L. Gosney, "Effect of exercise training on quality of life in multiple sclerosis: a meta-analysis," Mult Scler, vol. 14, pp. 129-35, 2008.

[2] U. Dalgas, E. Stenager, and T. Ingemann-Hansen, "Multiple sclerosis and physical exercise: recommendations for the application of resistance-, endurance- and combined training," Mult Scler, vol. 14, pp. 35-53, 2008.

[3] G. Kwakkel, R. C. Wagenaar, J. W. Twisk, G. J. Lankhorst, and J. C. Koetsier, "Intensity of leg and arm training after primary middlecerebral-artery stroke: a randomised trial," Lancet, vol. 354, pp. 1916, 1999.

[4] G. Kwakkel, B. J. Kollen, and H. I. Krebs, "Effects of robotassisted therapy on upper limb recovery after stroke: a systematic review," Neurorehabil Neural Repair, vol. 22, pp. 111-21, 2008.
[5] T. H. Massie and K. J. Salisburg, "The PHANTOM haptic interface: a device for probing virtual objects," Proceedings of the 1994 ASME International Mechanical Engineering Congress and Exhibition, pp. 295-302, 1994.

[6] E. G. Xydas and L. S. Louca, "Design and Development of a Haptic Peg-Board Exercise for the Rehabilitation of People with Multiple Sclerosis," EEE 10th International Conference on Rehabilitation Robotics. ICORR 2007., pp. 906-916, 2007.

[7] F. Amirabdollahian, G. T. Gomes, and G. Johnson, "The Peg-inHole: A VR-Based Haptic Assessment for Quantifying Upper Limb Performance and Skills," Proceedings of the 2005 IEEE 9th International Conference on Rehabilitation Robotics ThP01-22, pp. 422-425, 2005.

[8] J. De Boeck, G. Alders, D. Gijbels, T. De Weyer, C. Raymaekers, K. Coninx, and P. Feys, "The Learning Effect of Force Feedback Enabled Robotic Rehabilitation of the Upper Limbs in Persons with MS - a Pilot Study," Proceedings of 5th International Conference on Enactive Interfaces (ENACTIVE08), pp. 117-122, 2008.

[9] R. W. Bohannon, "Reference values for extremity muscle strength obtained by hand-held dynamometry from adults aged 20 to 79 years," Arch Phys Med Rehabil, vol. 78, pp. 26-32, 1997.

[10] D. E. Goodkin, D. Hertsgaard, and J. Seminary, "Upper extremity function in multiple sclerosis: improving assessment sensitivity with box-and-block and nine-hole peg tests," Arch Phys Med Rehabil, vol. 69, pp. 850-4, 1988.

[11] P. Feys, M. Duportail, D. Kos, P. Van Asch, and P. Ketelaer,

"Validity of the TEMPA for the measurement of upper limb function in multiple sclerosis," Clin Rehabil, vol. 16, pp. 166-73., 2002.

[12] L. Vanhees, J. Lefevre, R. Philippaerts, M. Martens, W.

Huygens, T. Troosters, and G. Beunen, "How to assess physical activity?," Eur J Cardiovasc Prev Rehabil, vol. 12, pp. 102-14, 2005. 